\title{
PREDIÇÃO DE QUALIDAdE DA MADEIRA E DA POLPA CELULÓSICA POR TÉCNICA DE ESPECTROSCOPIA DE INFRAVERMELHO PRÓXIMO (NIRS) ${ }^{1}$
}

\author{
Ricardo Balleirini dos Santos², José Lívio Gomide ${ }^{3}$ e Leonardo Chagas de Sousa²
}

\begin{abstract}
RESUMO-O objetivo deste estudo foi desenvolver modelos de calibração multivariada, com espectros infravermelhos próximos (NIR), para predição de qualidade da madeira e da polpa celulósica kraft de Eucalyptus. Foram utilizadas 30 amostras de madeira, em forma de cavacos, e 116 amostras de polpa kraft de Eucalyptus. Os cavacos foram utilizados com dois teores de umidade (33\% e 10\%). Nos cavacos mais úmidos, foram obtidas as seguintes correlações: $97 \%$ na densidade básica, $84 \%$ no teor de extrativos e $93 \%$ no teor de lignina. Nos cavacos mais secos, as correlações foram: $97 \%$ na densidade básica, $92 \%$ no teor de extrativos e $90 \%$ na lignina. Para a predição de qualidade das polpas, foram obtidos espectros NIR de folhas de celulose e das polpas na forma original (desagregada). Os modelos com espectros obtidos na folha de celulose apresentaram correlações de 94\% no número kappa, $93 \%$ na viscosidade da polpa, $90 \%$ no rendimento depurado e $86 \%$ no teor de ácido hexenurônico. Os modelos com espectros obtidos na polpa desagregada apresentaram as seguintes correlações: $90 \%$ no número kappa, $91 \%$ na viscosidade da polpa, $88 \%$ no rendimento depurado e $85 \%$ no teor de ácidos hexenurônicos. Os resultados demonstraram a viabilidade da aplicação da técnica de modelagem das características da madeira e da polpa celulósica, utilizando-se espectros NIR.
\end{abstract}

Palavras-chave: NIRS, madeira e polpa celulósica.

\section{PREDICTION OF WOOD AND KRAFT PULP QUALITY BY NEAR INFRARED SPECTROSCOPY (NIRS)}

\begin{abstract}
The objective of this study was to establish mathematical calibration models for near infrared spectra (NIRS) to predict wood quality and kraft pulp characteristics for Eucalyptus. Two different wood chip moisture contents were used (33\% and 10\%) and 116 kraft pulp samples were measured (kappa number 13-18). For more humid chips, the following correlations were established: $97 \%$ for wood basic density, $84 \%$ for extractive content and $93 \%$ for lignin content. For drier chips, the correlations were: $97 \%$ for wood basic density, $92 \%$ for extractive content and $90 \%$ for lignin content. For pulp quality prediction, NIRS spectra were obtained from handsheets and from the pulp in the original form (disaggregated). NIRS models for handsheets presented $94 \%$ correlation for kappa number, $93 \%$ for pulp viscosity, $90 \%$ for screened yield and $86 \%$ for hexenuronic acid content. Models for disaggregated pulp presented the following correlations: $90 \%$ for kappa number, $91 \%$ for pulp viscosity, $88 \%$ for screened yield and $85 \%$ for hexenuronic acid content. These results demonstrate the viability and precision of the NIRS technique to estimate wood quality and pulp characteristics.
\end{abstract}

Keywords: NIRS, wood and kraft pulp.

\footnotetext{
${ }^{1}$ Recebido em 28.09.2007 e aceito para publicação em 29.05.2009.

${ }^{2}$ Programa de Pós-Graduação em Ciência Florestal da Universidade Federal de Viçosa. E-mail: <ricardoballeirini @ yahoo.com.br>.

${ }^{3}$ Departamento de Engenharia Florestal da UFV. E-mail: <jlgomide@ufv.br>
} 


\section{INTRODUÇÃO}

O pujante desenvolvimento do setor nacional de celulose e papel tem sido possível pelo rápido crescimento das florestas de Eucalyptus e pela alta tecnologia empregada na produção de celulose. Essas condições possibilitam produção de polpa celulósica altamente competitiva no mercado mundial, inclusive com tradicionais produtores de celulose do Hemisfério Norte. A madeira de Eucalyptus, a principal matéria-prima utilizada pela indústria nacional, tem proporcionado produto final de alta qualidade e muito competitivo no mercado internacional.

Um fator importante na competitividade da indústria de celulose é a seleção de árvores para formação de florestas homogêneas que possibilitem a produção de polpa celulósica com alto rendimento industrial, baixo custo e elevada qualidade tecnológica. No Brasil, os programas de melhoramento florestal das empresas nacionais de celulose e papel se destacam no cenário mundial pelos excelentes resultados alcançados na área de clonagem de Eucalyptus. Os estudos de clonagem de Eucalyptus, para estabelecimento de florestas com elevada produtividade e alta qualidade, requerem análises e testes de milhares de clones potenciais. Apenas depois desses estudos silviculturais e tecnológicos é que são selecionados os clones para multiplicação e formação de florestas homogêneas que irão apresentar, após um período de rotação de 5 a 7 anos, alta produtividade florestal e, também, alta qualidade da madeira para produção de polpa celulósica (GOMIDE et al., 2004).

Para a caracterização da madeira e da polpa celulósica, visando à melhoria de qualidade da matériaprima e do produto final, são necessárias análises de rotina, que devem ser rápidas, precisas e de baixo custo. Os métodos laboratoriais disponíveis atualmente são demorados, caros e tediosos, o que os torna inviáveis para análises de grande número de amostras, impossibilitando, assim, um controle detalhado e preciso do processo.

Atualmente, no cenário internacional uma técnica que vem apresentando ótimo desempenho e viabilidade para a realização de análises da madeira e polpa celulósica é a espectroscopia de infravermelho próximo (NIRS).

A espectroscopia é uma técnica instrumental analítica que se baseia nas propriedades de absorção e emissão de energia eletromagnética das moléculas em regiões do espectro eletromagnético. No caso da espectroscopia
NIR, essa região localiza-se entre $700 \mathrm{~nm}$ e $2.500 \mathrm{~nm}$, e a resposta obtida é um sinal (espectro) interpretado por técnicas estatísticas de análises multivariadas dos dados. Se o espectrofotômetro infravermelho próximo (NIRS) for calibrado com precisão, é possível, pela análise dos espectros, predizer várias propriedades da madeira e da polpa celulósica. Essa calibração consiste em utilizar dados de análises laboratoriais convencionais e correlacionar os espectros infravermelhos próximos com as características da madeira ou da polpa.

Segundo Barathi et al. (2004), a técnica de análise da espectroscopia no infravermelho próximo tem sido muito empregada para a determinação de propriedades químicas da madeira e de polpas celulósicas, de forma rápida e precisa. Esses autores comprovaram a viabilidade dessa técnica para estabelecer características da polpa celulósica, realizando determinações "on-line" em processos industriais equipados com sensores de fibra ótica para a coleta dos espectros.

De acordo com Sparén et al. (2003), o número kappa de polpas pode ser predito, com sucesso, usando uma combinação de informações espectrais e modelos estatísticos multivariados. Esse sucesso está ligado à rapidez das leituras no aparelho NIRS, à fácil manipulação do sistema - incluindo a preparação de amostras - ao pequeno volume de amostras requerido na técnica e às altas correlações estatísticas alcançadas.

\section{MATERIAL E MÉTODOS}

Para a geração dos modelos de calibração do espectrofotômetro visando à caracterização tecnológica das madeiras, foram utilizadas amostras de clones de Eucalyptus provenientes do sul da Bahia. As amostras foram recebidas em forma de toras, que foram transformadas em cavacos em picador laboratorial dotado de três facas e duas peneiras classificadoras (GOMIDE et al., 2005).

As polpas celulósicas foram produzidas em laboratório, utilizando-se cavacos de 116 clones de Eucalyptus provenientes de diferentes regiões do Brasil.

\subsection{Construção dos modelos visando à caracterização tecnológica da madeira}

Para a construção dos modelos multivariados visando à predição da qualidade da madeira foram determinadas, primeiramente, as características 
tecnológicas das madeiras, incluindo densidade básica (TAPPI 258 om-85), teor de extrativos em álcool/tolueno (TAPPI, 1988) e teor de lignina klason (GOMIDE e DEMUNER, 1986). Os espectros NIR foram obtidos diretamente nos cavacos, em dois teores de umidade (33\% e 10\%), visando detectar a influência da umidade na calibração NIRS.

\subsection{Construção dos modelos para predição dos parâmetros de qualidade da polpa celulósica}

Foram realizados cozimentos kraft convencionais descontínuos ("batch"), empregando-se a técnica proposta por Gomide et al. (2004), para determinação das características de polpação em níveis de número kappa variando de 13 a 18 . Os outros parâmetros utilizados para a polpação kraft foram os seguintes, mantidos constantes para todos os cozimentos: cavacos $=250$ g a.s., sulfidez $=25 \%$, relação licor/ madeira $=4 / 1$, temperatura máxima $=171^{\circ} \mathrm{C}$, tempo até a temperatura máxima $=90$ min e tempo na temperatura máxima $=60 \mathrm{~min}$. Após a obtenção da polpa depurada, foram determinadas suas características tecnológicas, incluindo número kappa (TAPPI $236 \mathrm{~cm}-85$ ), viscosidade (TAPPI 230 om-89), rendimento depurado e teor de ácidos hexenurônicos (VUORINEM et al., 1996). Os espectros NIR foram determinados na polpa desagregada e na forma de folha de celulose.

\subsection{Calibração do NIRS}

O espectrofotômetro empregado para obtenção dos espectros na polpa e na madeira foi o NIRSystems 5000 da FOOS Tecator, que emite feixes de luz infravermelho próximos nos comprimentos de 1.100 a $2.500 \mathrm{~nm}$, em intervalos de $2 \mathrm{~nm}$.
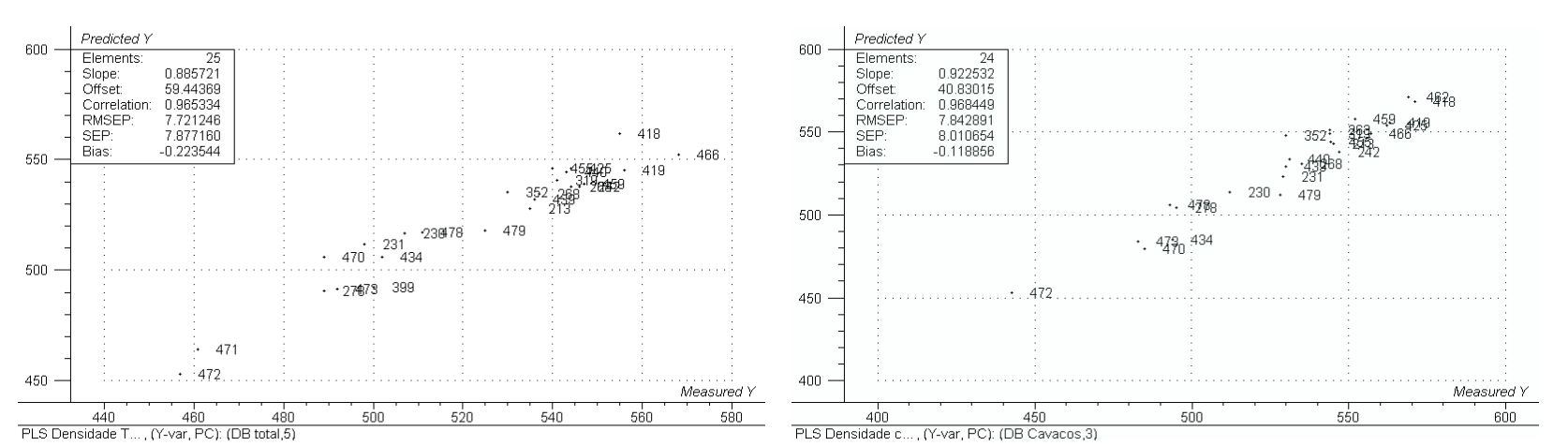

Figuras 1 e 2-Modelos de predição para densidade básica dos cavacos úmidos (33\% umidade) e secos (10\% umidade), respectivamente.

Figure 1 and 2 - Models for wood basic density using humid (33\% moisture) and dry (10\% moisture) chips, respectively.

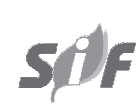

R. Árvore, Viçosa-MG, v.33, n.4, p.759-767, 2009
Os modelos matemáticos de calibração multivariada, tanto das madeiras quanto das polpas, foram desenvolvidos por meio da utilização do programa estatístico Unscrambler, versão 9.1, sendo os espectros obtidos submetidos a análises de componentes principais e análises de regressão pelo método de mínimos quadrados parciais (PLS).

Após a calibração, foram utilizados os métodos de validação cruzada e de validação externa para a análise da qualidade dos modelos.

\section{RESULTADOS E DISCUSSÃO}

\subsection{Modelos NIRS de caracterização tecnológica da madeira}

Nas Figuras 1 e 2 são apresentados os modelos de calibrações multivariadas desenvolvidos para a densidade básica da madeira nos teores de umidade de $33 \%$ e $10 \%$, respectivamente. No estabelecimento dos modelos multivariados foram retiradas, respectivamente, como "outliers", cinco e seis amostras nos dois teores de umidade. Neste estudo, as amostras foram consideradas "outliers" quando o seu espectro obtido apresentava comportamento inadequado e, ou, fugia do padrão das demais amostras. Foram obtidas correlações elevadas, de $97 \%$, e baixos erros médios de predição (RMSEP), de apenas 7,7 e $7,8 \mathrm{~kg} / \mathrm{m}^{3}$, respectivamente, dos dois teores de umidade. Esses resultados demonstram a alta precisão obtida na predição da densidade básica da madeira, utilizando diretamente os cavacos para obtenção de espectros NIR. O teor de umidade dos cavacos não apresentou diferença nas determinações, demonstrando que tanto cavacos com baixo teor de umidade (10\%) ou com umidade mais elevada (33\%) podem ser utilizados para obtenção dos espectros. 
Outra variável muito importante e passível de calibração utilizando espectros NIR é o teor de lignina Klason. O teor de lignina da madeira tem influência direta no consumo de reagentes químicos no processo de produção de celulose e, também, no rendimento do processo. Os modelos multivariados estabelecidos para o teor de lignina da madeira estão mostrados nas Figuras 3 e 4.

As correlações alcançadas nos modelos matemáticos para predição do teor de lignina diretamente nos cavacos foram de $93 \%$ e $91 \%$, com erros médios de predição de 0,6 e $0,7 \%$, respectivamente, dos dois teores de umidade. Esses resultados comprovam a alta precisão alcançada pela técnica NIRS na predição do teor de lignina em cavacos de madeira. Resultados semelhantes foram obtidos por Schultz (1990), que encontrou valores de correlação de $99 \%$, com erro médio de predição de $0,64 \%$, na determinação do teor de lignina por NIRS.
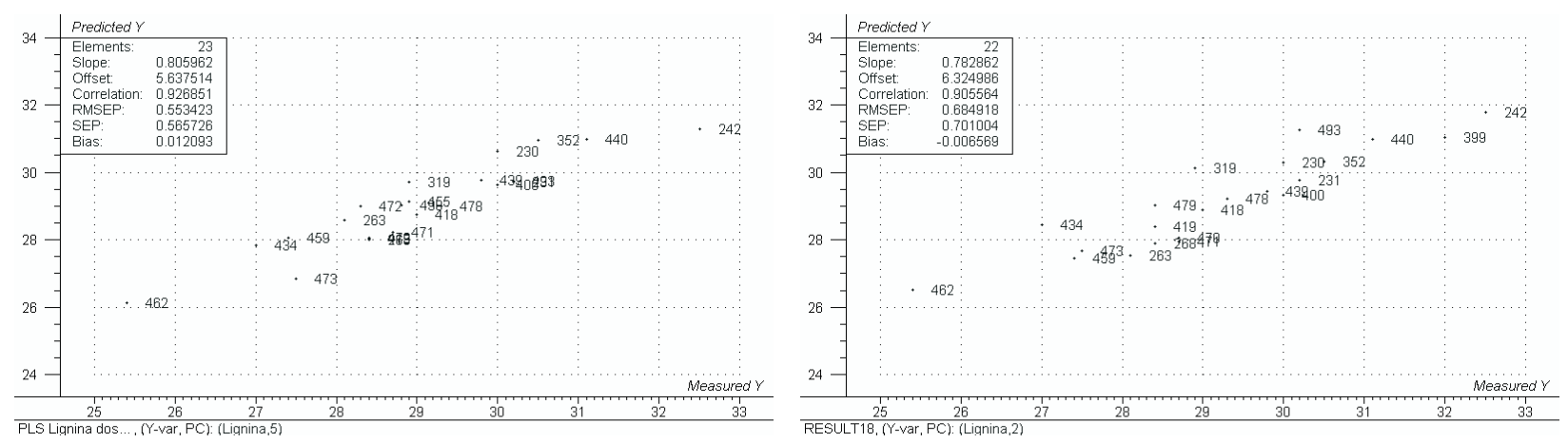

Figuras 3 e 4 - Modelos de predição para o teor de lignina Klason dos cavacos úmidos ( $33 \%$ umidade) e secos (10\% umidade), respectivamente.

Figure 3 and 4 - Models for Klason lignin content in humid (33\% moisture) and dry (10\% moisture) chips, respectively.
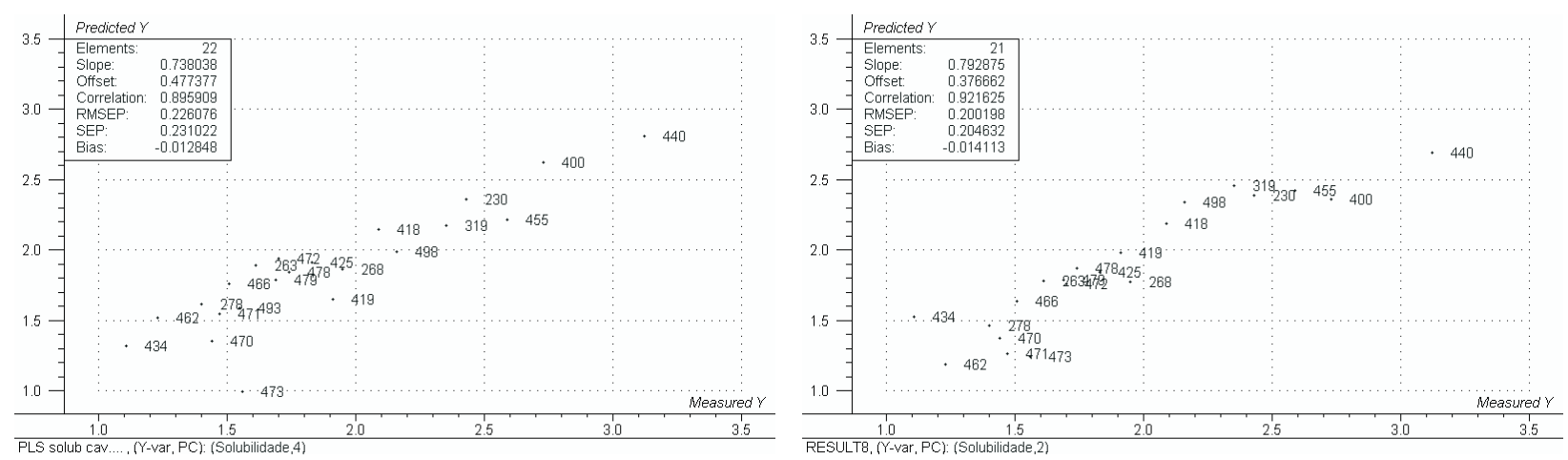

Figuras 5 e 6 - Modelos para teor de extrativos em álcool/tolueno utilizando cavacos úmidos (33\% umidade) e secos (10\% umidade), respectivamente.

Figure 5 and 6 - Models for extractives content in humid (33\% moisture) and dry (10\% moisture) chips, respectively.

R. Árvore, Viçosa-MG, v.33, n.4, p.759-767, 2009 
Nesse tipo de estudo, é importante ressaltar a quantidade de componentes principais utilizado na elaboração dos modelos de predição, sendo na madeira em forma de cavacos necessária a utilização de três componentes principais.

De modo geral, os modelos de predição para a madeira na forma de cavacos com $33 \%$ e $10 \%$ de umidade se mostraram semelhantes. No entanto, os modelos gerados para predição com cavacos úmidos foram um pouco mais satisfatórios pelo fato de terem apresentado menor quantidade de "outliers", sendo, portanto, mais precisos e representando, de forma mais ampla, o universo espectral estudado.

Os modelos gerados para predição de qualidade da madeira para celulose com a utilização de cavacos se mostraram precisos em termos de validação cruzada, como demonstrado pelo RMSEP nas Figuras 7 a 9. No entanto, é sempre necessária uma validação externa para comprovar a eficácia de modelos de predição. Por causa dos resultados ligeiramente melhores obtidos com os cavacos úmidos, a validação externa foi realizada para os modelos desses cavacos. Essa validação foi realizada com 15 amostras extras de cavacos de Eucalyptus que não participaram dos modelos multivariados.

Os resultados da validação externa para densidade básica (Figura 7) foram muito satisfatórios, com RMSEP (erro médio de predição) de $12 \mathrm{~kg} / \mathrm{m}^{3}$, demonstrando a precisão do modelo, uma vez que o erro médio foi próximo ao obtido na validação cruzada $\left(8 \mathrm{~kg} / \mathrm{m}^{3}\right)$.

As mesmas amostras utilizadas para a validação externa dos modelos para densidade básica foram também utilizadas para a validação dos modelos do teor de lignina Klason (Figura 8).

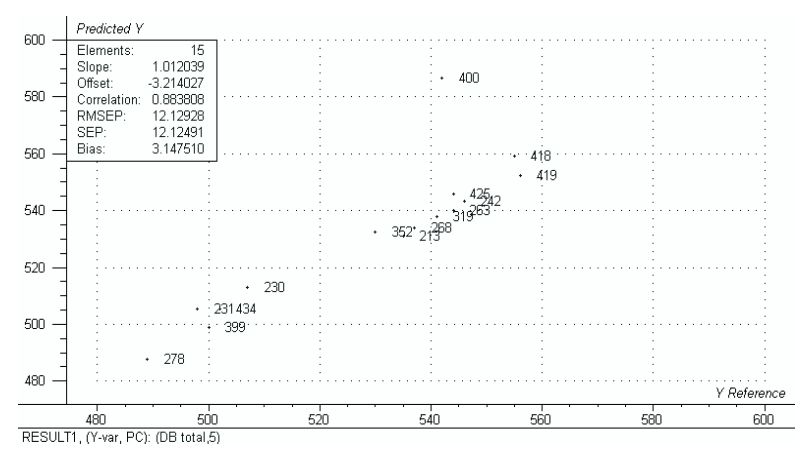

Figura 7 - Validação externa do modelo para densidade básica. Figure 7-External validation for wood basic density model.

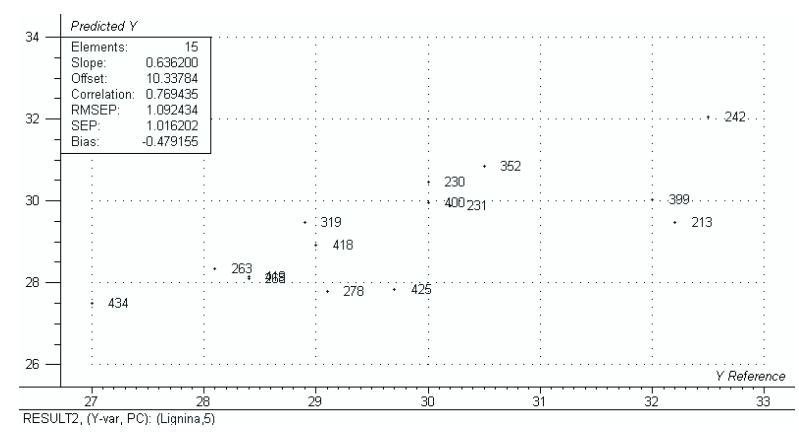

Figura 8 - Validação externa do modelo para o teor de lignina total.

Figure 8 - External validation for lignin content model.

O RMSEP da validação externa para o teor de lignina foi de $1,1 \%$, próximo do aceitável para determinações em laboratório, o que demonstra a possibilidade de utilização desse modelo para predição do teor de lignina em madeira na forma de cavacos pela técnica NIRS.

Na validação externa realizada para o teor de extrativos (Figura 9), o RMSEP foi de 0,65\%, podendo ser considerado bom, pois se aproxima do erro obtido em laboratório. A correlação foi próxima a $80 \%$ e demonstrou a viabilidade de utilização da técnica NIRS para predição do teor de extrativos em cavacos de Eucalyptus.

\subsection{Modelos de prediçãode parâmetros dequalidadedapolpa}

As características estatísticas dos modelos de calibração desenvolvidos para o número kappa, rendimento depurado, viscosidade e teor de ácidos hexenurônicos (AHex) das polpas celulósicas estão apresentadas nas Figuras 10 a 17 . Os modelos multivariados foram estabelecidos para as polpas celulósicas tanto na forma desagregada quanto na forma de folha.

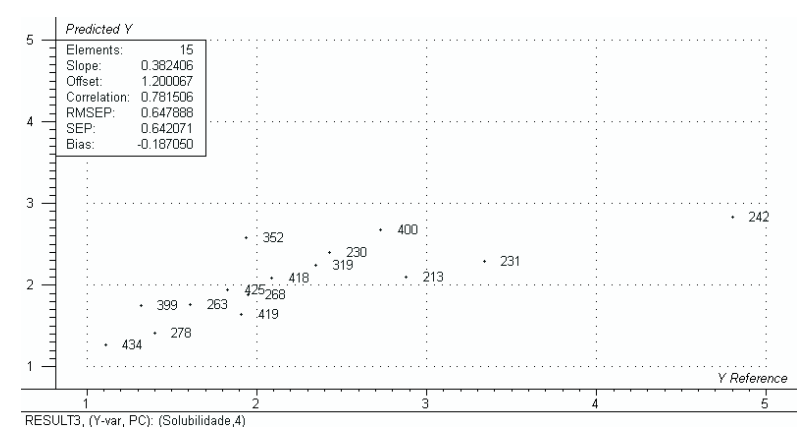

Figura 9-Validação externa do modelo para o teor extrativos. Figure 9 - External validation for extractives content model.

R. Árvore, Viçosa-MG, v.33, n.4, p.759-767, 2009 

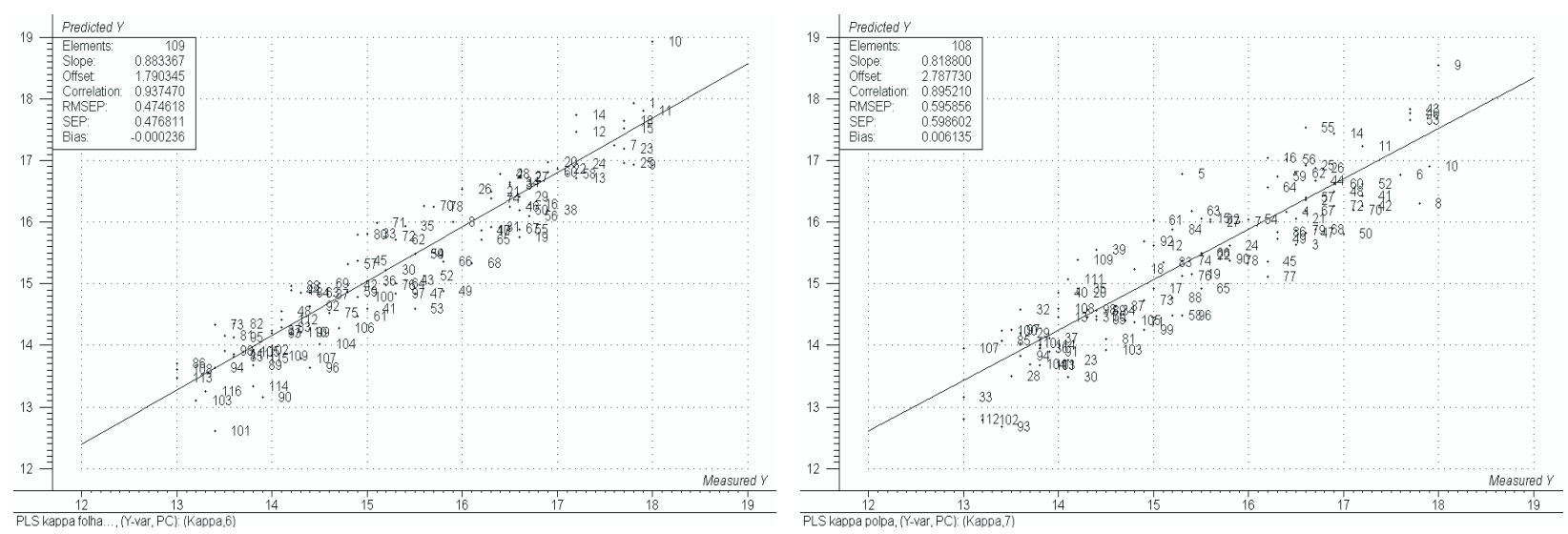

Figuras 10 e 11 - Modelos de predição do número kappa das polpas em forma de folha e desagregadas, respectivamente. Figure 10 and 11 -Models for kappa number prediction using handsheets and disaggregated pulp, respectively.
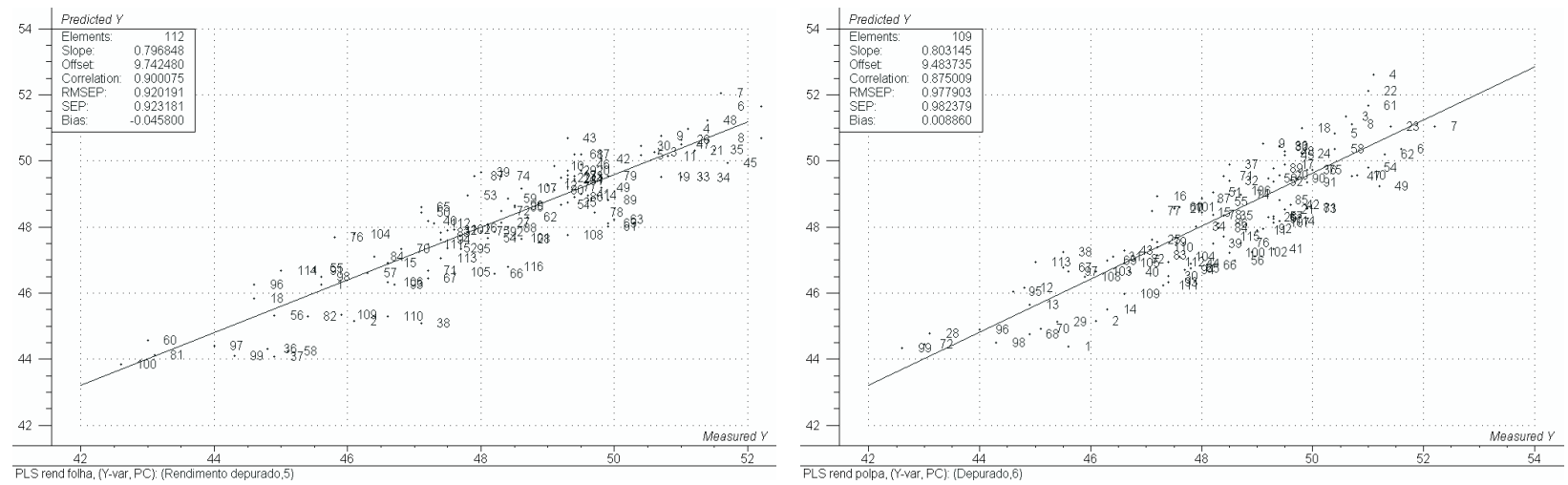

Figuras 12 e 13 - Modelos de predição do rendimento depurado utilizando, respectivamente, espectros obtidos na folha e na polpa desagregada.

Figure 12 and 13 - Models of screened yield prediction using handsheets and disaggregated pulp, respectively.
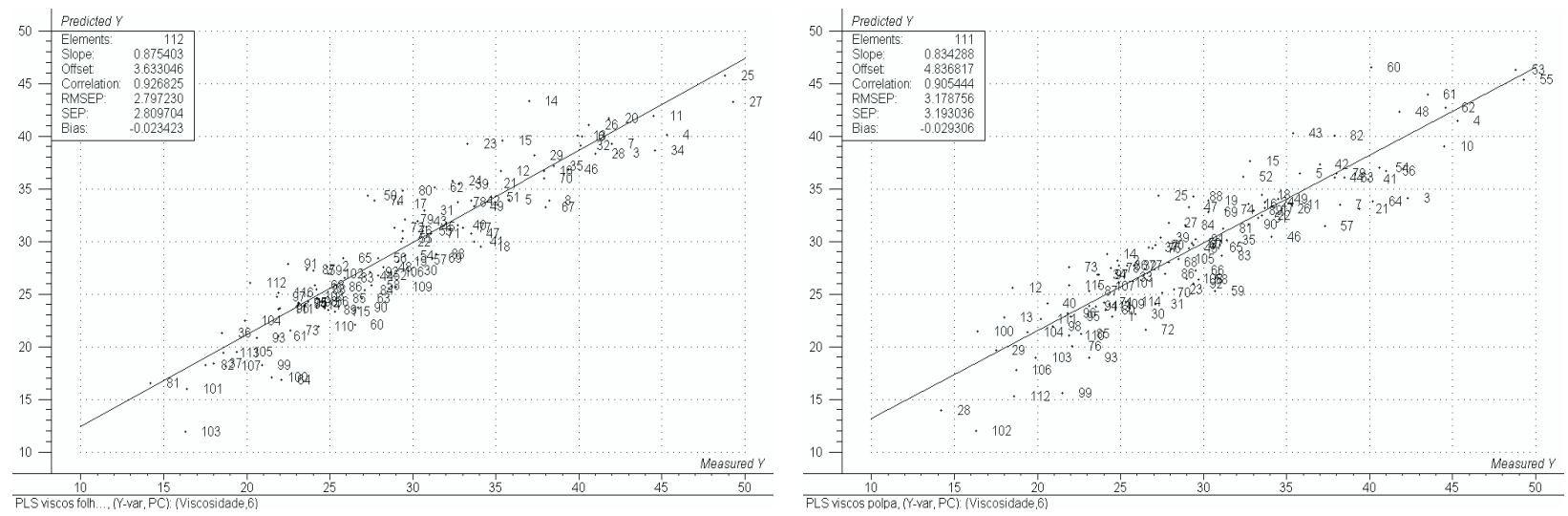

Figuras 14 e 15 - Modelos de predição da viscosidade utilizando, respectivamente, espectros obtidos na folha e na polpa desagregada.

Figure 14 and 15 - Models of viscosity prediction using handsheets and disaggregated pulp, respectively.

R. Árvore, Viçosa-MG, v.33, n.4, p.759-767, 2009 

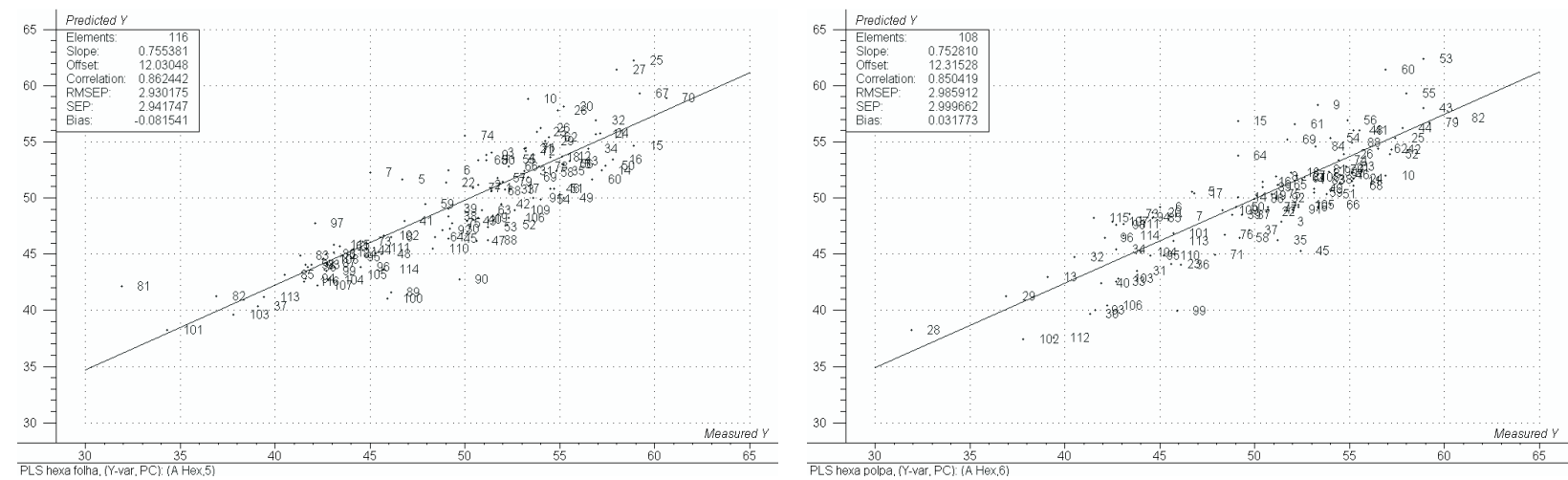

Figuras 16 e 17 - Modelos de predição do teor de ácidos hexenurônicos utilizando, respectivamente folha de celulose e celulose desagregada.

Figure 16 and 17 - Models for hexenuronic acids content using handsheets and disaggregated pulp, respectively.

A determinação do número kappa, uma análise de rotina nos laboratórios de celulose e papel, é uma operação relativamente demorada devido à necessidade de uniformização da amostra, secagem e reações químicas, justificando a utilização de técnicas alternativas mais rápidas como o NIRS, se considerada suficientemente precisa. Os modelos matemáticos NIRS desenvolvidos para predição do número kappa (Figuras 10 e 11) foram suficientemente precisos, com correlações de $94 \%$ e $90 \%$ e RMSEP de 0,5 e 0,6, respectivamente, nas polpas de celulose na forma desagregada e na forma de folha. Nesses modelos, os números de amostras consideradas "outliers" foram, respectivamente, 7 e 8 . Os modelos apresentaram, portanto, índices de precisão plenamente aceitáveis, demonstrando a validade de utilização dessa técnica para predição do número kappa. A utilização da polpa de celulose em forma de folha apresentou resultados com maior precisão.

O rendimento da polpação é um parâmetro de qualidade de grande importância, uma vez que essa característica tecnológica é consequência de vários fatores, englobando as características físicas, anatômicas e químicas da madeira e a demanda de álcali para a produção de polpa celulósica (GOMIDE et al., 2005). Como pode ser observado nas Figuras 12 e 13, as correlações obtidas nos modelos de predição do rendimento depurado foram satisfatórias (90\% e $88 \%)$, porém os RMSEP de ambas as determinações foram relativamente altos $(0,9 \%$ e $1,0 \%)$. Os números de "outliers" nos dois formatos de leituras NIRS foram 4 e 7 , demonstrando a maior precisão obtida para a celulose em forma de folha. Uma solução para melhoria desses modelos seria a utilização de maior número de amostras, possibilitando aumentar a precisão dos modelos e diminuir o RMSEP nos níveis mais confiáveis.

Os modelos para predição da viscosidade, mostrados nas Figuras 14 e 15, foram plenamente satisfatórios, demonstrando a possibilidade de utilização da técnica NIRS para determinação da viscosidade da polpa celulósica. As correlações obtidas foram elevadas (93\% e $91 \%)$, sendo os erros médios de predição baixos $(2,8$ e 3,2 cP) e o número de "outliers", também baixos (4 e 5), respectivamente, nos dois formatos de leitura NIRS.

Os modelos estabelecidos para a predição do teor dos ácidos hexenurônicos (Figuras 16e 17) foram, também, plenamente satisfatórios, indicando a viabilidade de utilização da técnica NIRS como alternativa para as análises convencionais. Caldeira et al. (2003) também demonstraram a eficiência da determinação do teor de ácidos hexenurônicos utilizando a técnica NIRS, tendo sido obtidos correlações acima de $90 \%$. Neste estudo, as correlações obtidas foram de $86 \%$ e $85 \%$ e os erros médios de predição, baixos, ou seja, de apenas 2,9 e $3,0 \mathrm{mmol} / \mathrm{kg}$, respectivamente, nas duas modalidades de leitura NIRS. No formato de folha, nenhuma amostra foi considerada "outlier", mas no formato desagregado oito amostras foram consideradas "outliers".

No caso da polpa celulósica, a quantidade de componentes principais utilizada na elaboração dos modelos de predição foi um pouco maior que na madeira na forma de cavacos, sendo necessários cinco componentes principais.

R. Árvore, Viçosa-MG, v.33, n.4, p.759-767, 2009 
Em todas as propriedades das polpas celulósicas estudadas, os modelos gerados a partir dos espectros obtidos na folha se apresentaram mais precisos em termos do número de amostras consideradas "outliers". Um dos fatores que podem ter contribuído para esses resultados poderia ser a maior desuniformidade da polpa celulósica na sua forma desagregada, em comparação com a folha de celulose. Essa desuniformidade resultaria em mais uma variável no conjunto de dados, e isso poderia resultar em modelos com menor capacidade de predição.

Mesmo com as boas correlações obtidas com a técnica de validação cruzada, conforme Figuras 10 a 17 , foram também realizados testes de validação externa para comprovar a precisão dos modelos. A validação externa foi realizada apenas nos modelos desenvolvidos nas folhas de celulose, uma vez que essa modalidade apresentou melhores resultados (correlações mais elevadas e menor número de "outliers"). A validação externa foi realizada com 29 amostras extras de polpa celulósica, produzidas nas mesmas condições de cozimento que as amostras utilizadas para a geração dos modelos.

Como observado na Figura 18, a correlação na validação externa para número kappa foi de $97 \%$, com RMSEP de 0,4. Esse resultado foi ainda melhor que as predições obtidas na validação cruzada para geração dos modelos de calibração. Tal fato não é comum, mas pode ser explicado pela uniformidade das amostras estudadas, pela qualidade dos cozimentos e pela precisão da técnica de determinação do número kappa.

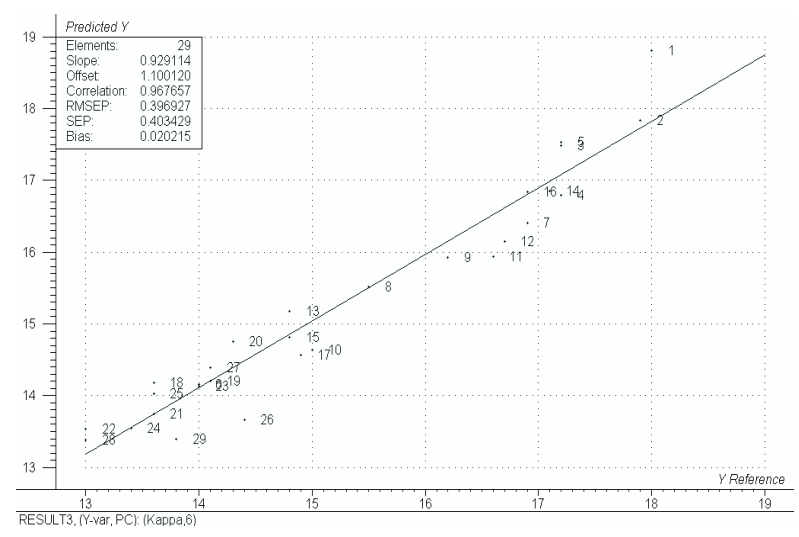

Figura 18 - Validação externa do modelo para determinação do número kappa.

Figure 18 - External validation for kappa number.
Como pode ser observado na Figura 19, a correlação na validação externa para rendimento depurado foi de $89 \%$, com RMSEP de $0,9 \%$. Esses resultados da validação externa comprovam a precisão do modelo e indicam a possibilidade de sua utilização, sendo, entretanto, aconselhável uma melhoria do RMSEP a ser obtida com inclusão de novas amostras no modelo.

Na validação externa do modelo multivariado da viscosidade, a correlação encontrada foi de $95 \%$, com RMSEP de 2,4 cP (Figura 20), confirmando a precisão do modelo e demonstrando a possibilidade de sua utilização como alternativa ao método convencional de laboratório.

A correlação obtida na validação externa do teor de ácidos hexenurônicos foi de $95 \%$ e o erro médio de predição, de $1,9 \mathrm{mmol} / \mathrm{kg}$ (Figura 21), o que demonstra a alta qualidade do modelo gerado e a possibilidade de seu uso para predição dessa variável, com alta precisão em relação ao método convencional de análise.

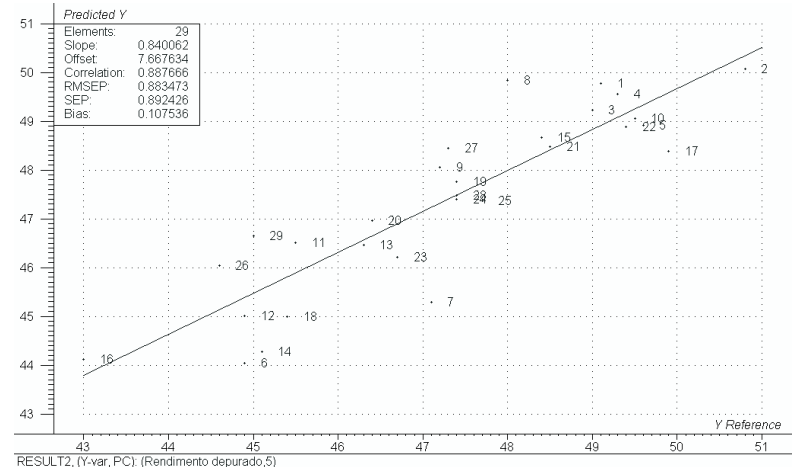

Figura 19 - Validação externa da determinação do rendimento depurado.

Figure 19 - External validation for screened yield.

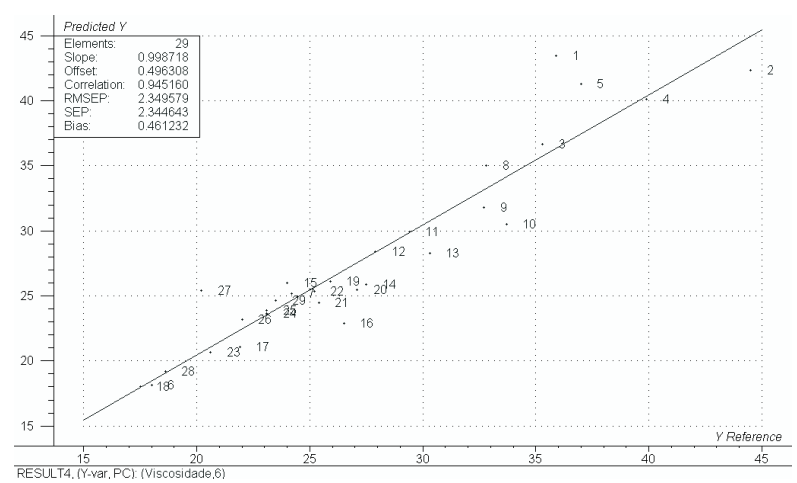

Figura 20 - Validação externa da determinação de viscosidade. Figure 20 - External validation for pulp viscosity. 


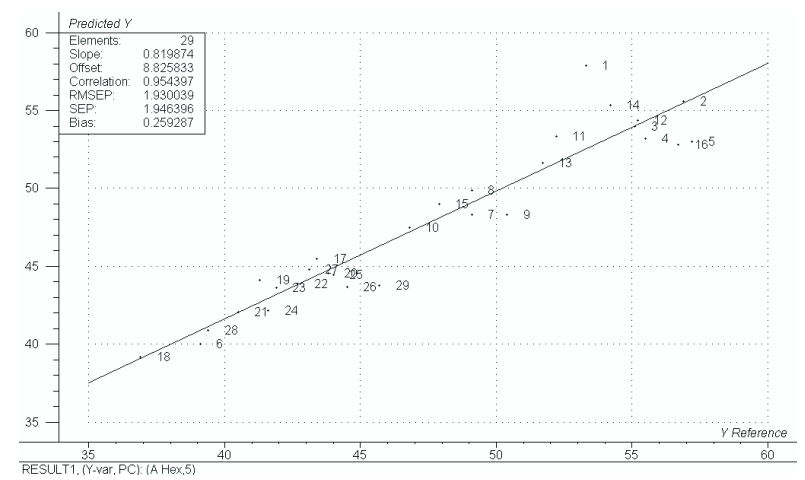

Figura 21 - Validação externa para o teor de ácidos hexenurônicos.

Figure 21 - External validation for hexenuronic acids content.

\section{CONCLUSÕES}

Os resultados deste estudo permitiram concluir que:

- A utilização do aparelho NIRSystems 5000 da FOSS é perfeitamente viável para a aquisição dos espectros diretamente nos cavacos e na polpa celulósica.

- Teor de umidade mais alto dos cavacos (33\%) proporcionou resultados melhores.

- Os modelos gerados para densidade básica, teor de lignina total e teor de extrativos apresentaram altas correlações e baixos erros médios de predição.

- Os modelos gerados com espectros coletados nas folhas de celulose apresentaram melhores correlações e menores erros médios de predição, em relação aos obtidos com a polpa celulósica na forma desagregada.

- Os modelos gerados para número kappa e viscosidade das polpas mostraram correlações acima de $90 \%$ e erros médios de predição dentro de limites aceitáveis em laboratório.

- Os modelos para ácidos hexenurônicos exibiram correlações próximas a $90 \%$, com baixo erro médio de predição de apenas $3,0 \mathrm{mmol} / \mathrm{kg}$.

- Para o modelo de predição de rendimento depurado, novas amostras deveriam ser acrescentadas para possibilitar diminuição do erro médio de predição, uma vez que o valor obtido se apresentou acima do aceitável em análises laboratoriais.

\section{REFERÊNCIAS}

BARATHI, M. H.; MacGREGOR, J. F.; CHAMPAGNE, M. Using near-infrared multivariate image regression to predict pulp properties. Tappi Journal, v.3, n.5, p.8-13, 2004.

CALDEIRA, A. F.; SANTOS, S. L.; SACON, V. Hexenuronic acid, klason lignin and viscosity of pulp predicted by NIR spectroscopy. In: INTERNATIONAL COLLOQUIUM ON EUCALYPTUS KRAFT PULP, 2003, Viçosa, MG. Anais... Viçosa, MG: UFV, 2003. p.151-160.

GOMIDE, J. L.; DEMUNER, B. J. Determinação do teor de lignina em material lenhoso: Método Klason modificado.O Papel, v.47, n.8, p.36-38, 1986.

GOMIDE, J. L. et al. Caracterização tecnológica, para produção de celulose, da nova geração de clones de Eucalyptus do Brasil. Revista Árvore, v.29, n. 1, p.129-137, 2005.

GOMIDE, J. L.; FANTUZZI NETO, H.; LEITE, H. G. Estratégia de análise da qualidade de madeira de Eucalyptus sp. para produção de celulose. Revista Árvore, v.28, n.3, p.443-450, 2004.

SCHULTZ, T. P.; BURNS, D. A. Rapid secondary analysis of lignocellulose: comparison of near infrared and fourier transform infrared. Tappi Journal, v.73, n.5, p.209-212, 1990.

SPARÉN, A. et al. Determination of kappa number reduction in pulp and COD in bleach plant effluents using multiwavelength spectrometry and multivariate calibration. Tappi Journal, v.2, n.1, p.16-21, 2003.

\section{TECHNICALASSOCIATION OF PULP \& PAPER INDUSTRY. Tappi test methods 2000-2001. Atlanta: Tappi Press, 2000.}

VUORINEM, T. et al. Selective hydrolysis of hexenuronic acid groups and its application in ECF and bleaching of kraft pulps. In: INTERNATIONALPULPBLEACHING CONFERENCE, 1996, Washington, D.C. Anais... Washington: CCD, 1996. p.15.

R. Árvore, Viçosa-MG, v.33, n.4, p.759-767, 2009 